\title{
One-pot facile synthesis of nanorice-like structured CuS@WS as an advanced electroactive material for high-performance supercapacitors
}

\author{
Atluru Lakshmi Prasanna ${ }^{1}$ Kummara Venkata Guru Raghavendra ${ }^{1}$ P. Himasree ${ }^{1}$. Ikkurthi Kanaka Durga ${ }^{1}$. \\ Chandu V. V. Muralee Gopi ${ }^{2} \cdot$ S. Srinivasa Rao ${ }^{3} \cdot$ Hee-Je Kim ${ }^{1}$
}

Received: 16 December 2019 / Accepted: 7 February 2020 / Published online: 14 February 2020

(c) Springer Nature Switzerland AG 2020

\begin{abstract}
Binder-free nanorice-like featured CuS@WS 2 structures have been synthesized using a simple and cost-effective chemical bath deposition approach and their application as electroactive material for high-performance supercapacitors. The surface properties of morphology, structure and composition of the as-prepared electrodes are examined using the scanning electron microscopy, transmission electron microscopy, X-ray diffraction and X-ray photoelectron spectroscopy, respectively. The nanorice-like featured CuS@WS 2 electrode exhibits nanorice-like structures, which provides the abundant active sites for redox reactions and facilitates the electrolyte diffusion. The electrochemical performance of the supercapacitor electrodes was examined by cyclic voltammetry and galvanostatic charge-discharge studies. From the electrochemical tests, the CuS@WS 2 electrode exhibits a higher specific capacitance $\left(C_{\mathrm{s}}\right)$ of $887.15 \mathrm{~F} \mathrm{~g}^{-1}$ at a current density of $3.75 \mathrm{~A} \mathrm{~g} \mathrm{~g}^{-1}$ with greater energy density and excellent rate capability compared to bare CuS $\left(588.0 \mathrm{~F} \mathrm{~g}^{-1}\right)$ and

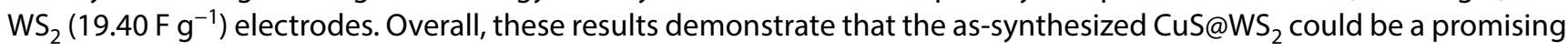
material for next-generation high-performance electrochemical energy storage applications.
\end{abstract}

Keywords CuS@WS $\cdot$ Nanorice-like·Chemical bath deposition method·Supercapacitors·Specific capacitance

\section{Introduction}

The huge demand of the energy consumption made fossil fuels to perish earlier which enabled to rely on the most feasible energy sources such as renewable energy sources. Numerous experiments were conducted to store this energy and eventually found the more efficient energy storage devices such as supercapacitors, batteries, etc. [1-3]. These supercapacitors have some appreciable characteristics such as fast-charging capabilities, greater power densities and longer life period. Hence, they are also termed as electrochemical capacitors that have more industrial and technical applications [4,5]. Depending on their charge storage phenomenon, the supercapacitors are categorized into two types such as (1) electrical double-layer supercapacitors, where the capacitance occurs because of the ion adsorption/desorption in between the carbon electrode and redox electrolyte, and (2) Faraday (redox) supercapacitors, where the higher capacitances are observed at reversible faster redox reactions.

EDLC supercapacitor electrodes are widely fabricated using the porous carbon materials. At the same time, the

Atluru Lakshmi Prasanna and Kummara Venkata Guru Raghavendra have equally contributed to this work.

$\triangle$ Hee-Je Kim, heeje@pusan.ac.kr|'School of Electrical Engineering, Pusan National University, Busandaehak-ro 63beon-gil, Geumjeong-gu, Busan 46241, South Korea. ${ }^{2}$ Photonics Laboratory, Division of Computer, Electrical, and Mathematical Sciences and Engineering, King Abdullah University of Science and Technology (KAUST), Thuwal Jeddah 23955-6900, Saudi Arabia. ${ }^{3}$ Department of Mechatronics Engineering, Kyungsung University, 309 Suyeong-ro, Nam-gu, Busan 48434, Korea. 
pseudocapacitor electrodes are made from transition metal oxides, hydroxides, sulfides and conducting polymers, etc. For various applications in solar cells, supercapacitors and photocatalysis [6-10]. In practical applications such as electric vehicles, there are some limitations when using the high-cost transition metal oxides $\left(\mathrm{RuO}_{2}\right.$ and $\left.\mathrm{V}_{2} \mathrm{O}_{5}\right)[11,12]$. Hence, there is a greater importance to fabricate pseudocapacitors with low-cost materials. There are some reports in recent time that suggest mixing two metal oxides increases the specific capacitance and results in greater electrical conductivity [13].

Because of their good electrochemical abilities, transition metal sulfides (TMS) were found to be the good option for electrode materials in supercapacitor applications [14]. Different TMS such as $\operatorname{CoS}_{x}, \mathrm{Ni}_{3} \mathrm{~S}_{2}$ and $\mathrm{CuS}$ can be used as electroactive materials for supercapacitor applications [15-17]. Tungsten disulfide $\left(\mathrm{WS}_{2}\right)$ is one of the typical layered transition metal dichalcogenide (TMD) has found to be the efficient SC electrode material observed from its good layered structure and excellent conductivity [18-20]. Higher capacitive values can be achieved when used as nanostructured materials than bulk materials. Further, this exhibits an enhanced surface area and minimal transfer channels for ions/electrons resulting in good capacitance [21]. Though CuS has a greater usage in supercapacitor applications, there is a huge research going on the $\mathrm{WS}_{2}$ to identify the electrochemical capacitance properties of nanostructured $\mathrm{WS}_{2}[22,23]$. Tu et al. synthesized $\mathrm{WS}_{2}$ nanostructures and showed a specific capacitance of $398.5 \mathrm{~F} \mathrm{~g}^{-1}$ for sheet-like $\mathrm{WS}_{2}$ structures [24]. Pan et al. [25] synthesized a CuS-decorated Ti3C2 MXene for asymmetric supercapacitors and obtained a specific capacitance of $169.5 \mathrm{C} \mathrm{g}^{-1}$ at $1 \mathrm{~A} \mathrm{~g}^{-1}$. Kumar et al. [26] fabricated the $\mathrm{CuS} / \mathrm{Cu}(\mathrm{OH})_{2}$ nanocomposite material for supercapacitor applications and achieved the $845.5 \mathrm{~F} / \mathrm{g}$ at $1 \mathrm{~mA} / \mathrm{cm}^{2}$. Raghavendra et al. [27] synthesized the CuS electrode and obtained the specific capacity of $164.053 \mathrm{~m} \mathrm{Ah} \mathrm{g}^{-1}$ at $1 \mathrm{~A} \mathrm{~g}^{-1}$.

Inspired from the above works, the present work reports the method of chemical bath deposition for the synthesis of nanorice-like structured CuS@WS 2 that is used for various supercapacitor applications. Further, this $\mathrm{CBD}$ method is very simple to operate, low cost and can be implemented for deposition of large area. This paper includes the synthesis of nanorice-like structured CuS@ $\mathrm{WS}_{2}$ electrode was prepared by CBD on the surface of $\mathrm{Ni}$ foam substrate and that can be employed as supercapacitor electrode material. The electrochemical studies were analyzed through cyclic voltammetry (CV) and galvanostatic charge-discharge measurements in a $3 \mathrm{M}$ $\mathrm{KOH}$ solution using three-electrode setup. The formation of a nanorice-like structured CuS@WS 2 gives rise to better electrical conductivity, faster electrolyte diffusion and rapid electron pathways compared to those of bare CuS and $\mathrm{WS}_{2}$ electrode materials. As a result, the nanorice-like structuredCuS@WS 2 electrode exhibits a higher specific capacitance than the bare $\mathrm{CuS}$ and $\mathrm{WS}_{2}$ electrodes.

\section{Experimental}

\subsection{Materials}

All the chemicals used in the synthesis were of analytical grade purchased from Sigma-Aldrich. Copper sulfate $\left(\mathrm{CuSO}_{4}\right)$, urea $\left(\mathrm{CH}_{4} \mathrm{~N}_{2} \mathrm{O}\right)$, sodium thiosulfate $\left(\mathrm{Na}_{2} \mathrm{~S}_{2} \mathrm{O}_{3}\right)$, sodium tungstate dihydrate $\left(\mathrm{Na}_{2} \mathrm{WO}_{4}\right)$ were used as obtained.

\subsection{Preparation of CuS, $\mathrm{WS}_{2}$ and CuS@WS electrodes}

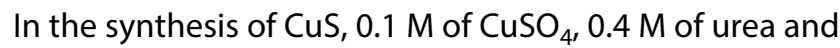
$0.4 \mathrm{M}$ of $\mathrm{Na}_{2} \mathrm{~S}_{2} \mathrm{O}_{3}$ were dissolved in $70 \mathrm{~mL}$ of deionized (DI) water and constantly stirred for $30 \mathrm{~min}$. Nickel (Ni) foams (of size $2 \times 5 \mathrm{~cm}^{2}$ ) cleaned by ultrasonication process were dipped in the as-prepared CuS solution and kept in the oven at $90^{\circ} \mathrm{C}$ for $6 \mathrm{~h}$. After cooling to room temperature, the Ni foams were further washed with ethanol, DI water, dried in over at $60^{\circ} \mathrm{C}$ for overnight and stored properly.

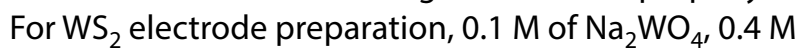
of urea and $0.4 \mathrm{M}$ of $\mathrm{Na}_{2} \mathrm{~S}_{2} \mathrm{O}_{3}$ were dissolved in $70 \mathrm{~mL}$ of DI water. The ultrasonically cleaned $\mathrm{Ni}$ foams were immersed in the as-prepared $\mathrm{WS}_{2}$ solution and kept in hot-air oven for $90^{\circ} \mathrm{C}$ for $6 \mathrm{~h}$. Further, the obtained electrodes were washed properly and dried in the oven at $60^{\circ} \mathrm{C}$ for $12 \mathrm{~h}$ and used for electrochemical measurement.

In the synthesis of CuS@WS $2,0.1 \mathrm{M}$ of $\mathrm{CuSO}_{4}, 0.4 \mathrm{M}$ of urea and $0.4 \mathrm{M}$ of $\mathrm{Na}_{2} \mathrm{~S}_{2} \mathrm{O}_{3}, 0.1 \mathrm{M}$ of $\mathrm{Na}_{2} \mathrm{WO}_{4}$ were dissolved in $70 \mathrm{~mL}$ of DI water and further stirred for $30 \mathrm{~min}$ to make a constant homogenous solution. Then, $\mathrm{Ni}$ foams were immersed in this solution and kept in oven at $90^{\circ} \mathrm{C}$ for $6 \mathrm{~h}$. After this, the Ni foams were washed with ethanol and DI water and dried in the oven at $90^{\circ} \mathrm{C}$ for $12 \mathrm{~h}$ and stored properly for the characterizations.

\subsection{Characterization}

The electrode morphology can be characterized by field emission scanning electron microscopy (FE-SEM S2400, Hitachi). Transmission electron microscopy (TEM). X-ray photoelectron spectroscopy (XPS, KBSI, Busan) was performed to examine the chemical states of the elements. The phase structure of the copper sulfide-based electrode was analyzed by X-ray diffraction (XRD, D/Max-2400, Rigaku) using Cu ka source. 


\subsection{Electrochemical measurements}

The electrochemical measurements were carried out using BioLogic-SP150 workstation, and the experimental tests were performed using a three-electrode setup with CuS@ $\mathrm{WS}_{2}$ as the working electrode, $\mathrm{Ag} / \mathrm{AgCl}$ as the reference electrode and $\mathrm{Pt}$ wire as the counter electrode. The aqueous $3 \mathrm{M}$ $\mathrm{KOH}$ is used as the electrolyte. The specific capacitance $\left(C_{s}\right.$, $\left.\mathrm{F} \mathrm{g}^{-1}\right)$, energy density $\left(E, \mathrm{Wh} \mathrm{kg}^{-1}\right)$ and power density $(P$, $\mathrm{W} \mathrm{kg}^{-1}$ ) of the as-prepared electrodes were calculated using the following equations:

$C_{\mathrm{s}}=\frac{I \times \Delta t}{m \times \Delta V}$

$E=\frac{0.5 \times C_{\mathrm{s}} \times \Delta V^{2}}{3.6}$

$$
P=\frac{E \times 3600}{\Delta t}
$$

where $I, \Delta t, \Delta V$ and $m$ represent the current (A), discharge time (s), potential window $(\mathrm{V})$ and mass $(\mathrm{g})$ of the electroactive material, respectively.

\section{Results and discussion}

The CuS, WS 2 and CuS@WS 2 electrode materials were grown on Ni foam surface using facile chemical bath deposition method. SEM and TEM analysis were performed to study the morphologies of the electrodes under a variety of magnifications, as shown in Fig. 1. From Fig. 1a, the CuS nanoparticles were grown steadily on the surface of the Ni foam substrate. In Fig. 1a1, a2, every nanoparticle was composed of the very small tiny bundle of widespread nanorice-like particles. Further, it is observed that from
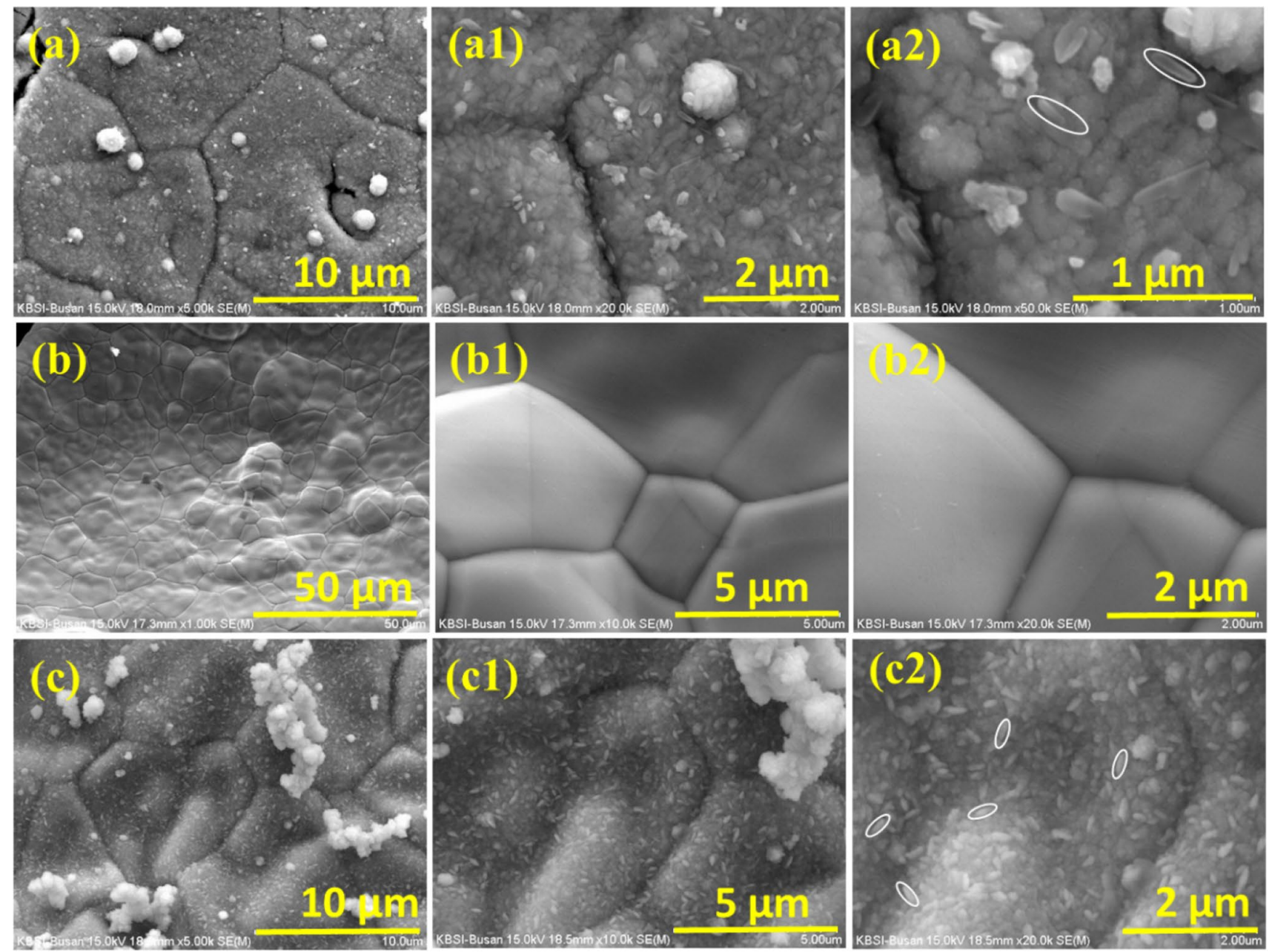

Fig. 1 Low- and high-magnification SEM images of (a, a1, a2) CuS, (b, b1, b2) WS ${ }_{2}$ and (c, c1, c2) composite CuS@WS 2 electrodes on Ni foam surface 
Fig. $1 b, b 1, b 2$ that the smooth surface of agglomerated nanosheets was covered over the Ni foam surface homogenously exhibiting an intermediate resistance of WS2 electrode. Concurrently, Fig. 1c, c1, c2 shows that the nanoricelike particles were distributed on the Ni foam.

The structure of as-prepared material on $\mathrm{Ni}$ foam was further confirmed by TEM characterization. Figure 2 depicts the TEM images of the as-prepared CuS, $\mathrm{WS}_{2}$ and CuS@WS 2 electrodes, respectively. The TEM image of CuS material exhibits the agglomerated nanoparticle structures (Fig. 2a, a1). Figure 2b, b1 shows the TEM image of nanosheet-like structures. The TEM image in Fig. $2 c, c 1$ represents the homogenous distribution of nanorice-like structures which were interconnected with each other. The TEM results are consistent with the SEM studies. The observed unique surface area and unique structure are advantageous to electrolyte diffusion and electron transfer, which promotes the electrochemical activity of CuS@ $\mathrm{WS}_{2}$.

XRD analysis was performed to obtain the crystal structures and phase purity of CuS, WS ${ }_{2}$ and CuS@WS 2 precursors as shown in Fig. 3. The XRD peaks for CuS observed at $21.78^{\circ}, 27.71^{\circ}, 31.82^{\circ}, 35.05^{\circ}, 43.14^{\circ}$ and $53.27^{\circ} 2 \theta$ were given to the (0 04$),\left(\begin{array}{lll}1 & 0 & 1\end{array}\right),\left(\begin{array}{lll}1 & 0 & 3\end{array}\right),(104),(106)$, and (1 14 ) planes of the CuS peaks (JCPDS No: 01-079-2321), respectively [28]. For $\mathrm{WS}_{2}$, the peaks observed at $14.3^{\circ}, 32.7^{\circ}$, $39.5^{\circ}, 57.3^{\circ}, 60.0^{\circ}, 70.1^{\circ}$ and $78.3^{\circ}$ were given to the $(00$

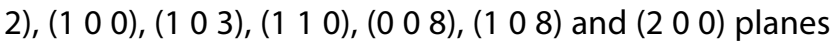
of the hexagonal-phase $\mathrm{WS}_{2}$ structure (JCPDS 84-1398), respectively [29].

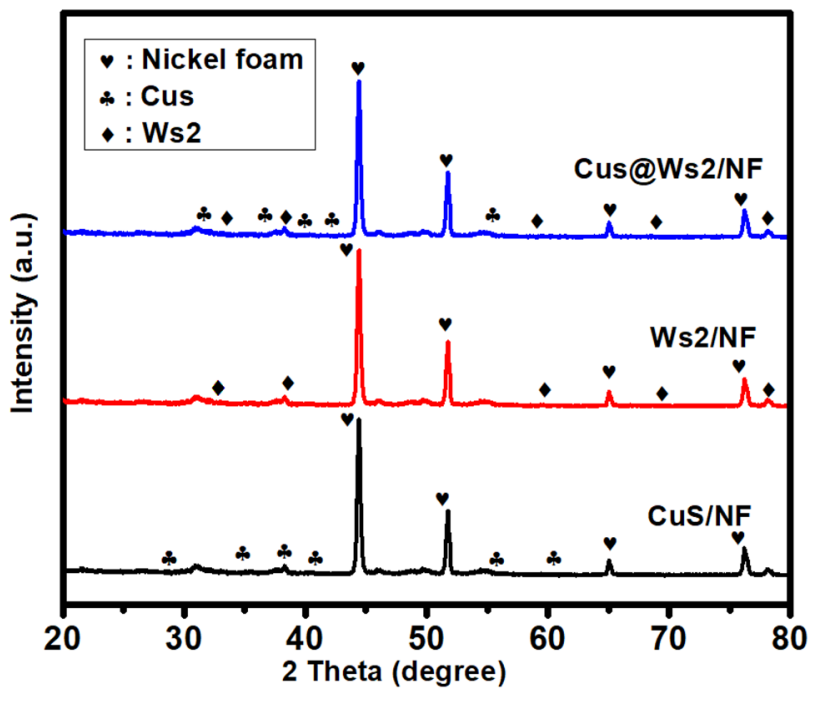

Fig. 3 XRD pattern of CuS, WS 2 and composite CuS@WS 2 electrode on Ni foam

XPS was performed to find the chemical composition and the oxidation states of the various compounds like CuS, WS 2 , CuS@WS . Figure 4a shows the XPS survey spectrum of the CuS, WS ${ }_{2}, \mathrm{CuS}_{\mathrm{O}} \mathrm{WS}_{2}$ electroactive materials on $\mathrm{Ni}$ foam. The bare CuS and $\mathrm{WS}_{2}$ survey scans show the existence of $\mathrm{Cu}$ and $\mathrm{S}$ and $\mathrm{W}$ and $\mathrm{S}$ signals. More interestingly, the composite of CuS@WS survey spectrum shows the presence of the $\mathrm{Cu}, \mathrm{W}$ and $S$ elements. The Ni peak in the XPS survey spectrum is
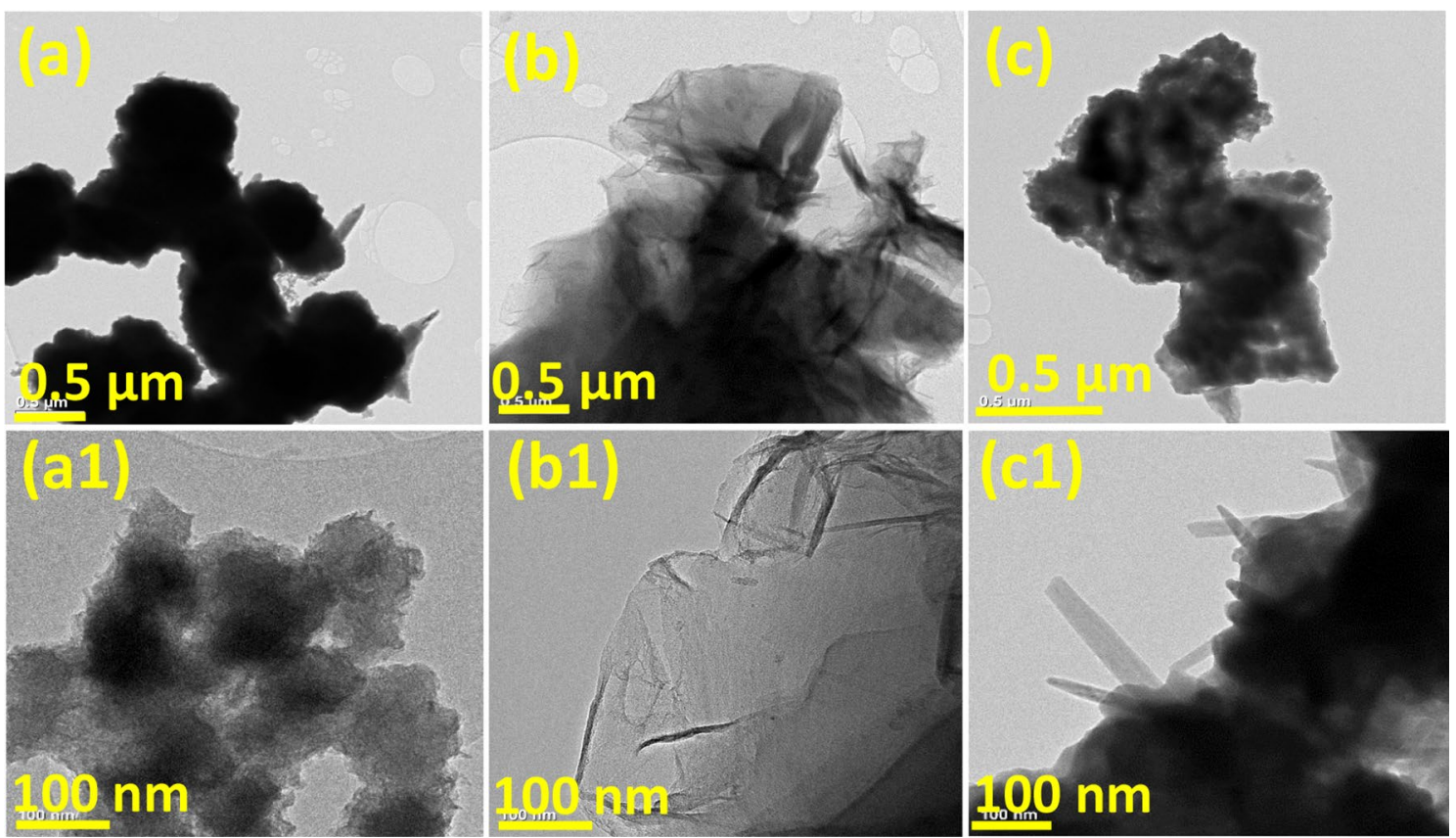

Fig. 2 TEM images of (a, a 1) CuS, (b, b1) WS 2 and (c, c1) CuS@WS ${ }_{2}$ electrodes

\section{SN Applied Sciences}



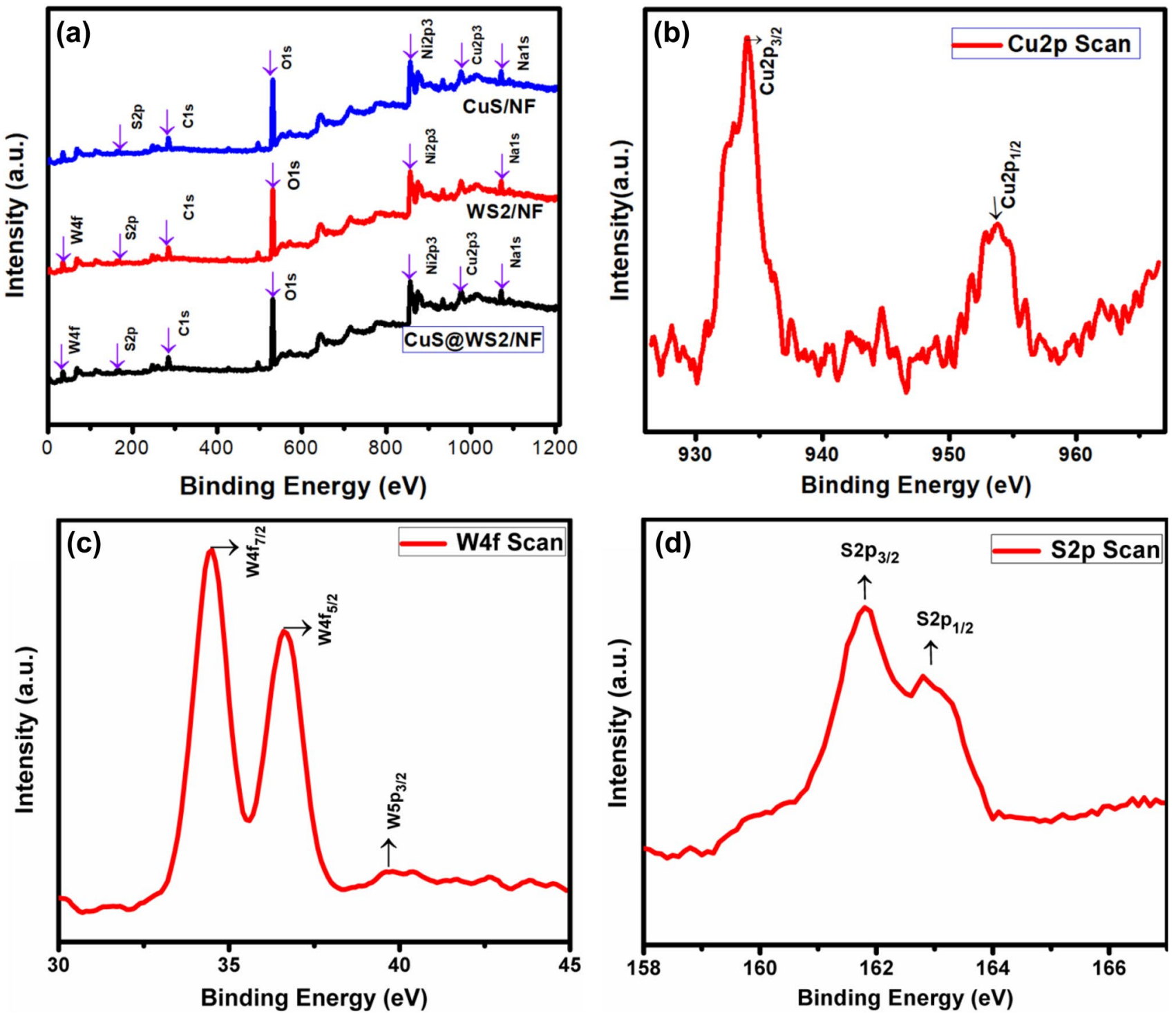

Fig. 4 a XPS survey spectra of as-prepared CuS, WS ${ }_{2}$ and CuS@WS 2 electrodes on Ni foam. High-resolution XPS spectra of b Cu2p, c W4f and d S2 $p$ signals in CuS@WS 2 electrodes

due to the background of $\mathrm{Ni}$ foam. Figure $4 \mathrm{~b}$ shows the high-resolution $\mathrm{Cu} 2 p$ spectrum. The $\mathrm{Cu} 2 p$ spectrum exhibits the two strong peaks at the binding energies of 934.0 and $953.87 \mathrm{eV}$, which are assigned to $\mathrm{Cu} 2 p_{3 / 2}$ and $\mathrm{Cu} 2 p_{1 / 2}$, respectively [30]. The difference in binding energy of these two peaks was approximately $19.87 \mathrm{eV}$. These peaks can be ascribed to the $\mathrm{Cu}^{2+}$ state in CuS structures. Figure $4 c$ depicts the high-resolution XPS spectrum of $\mathrm{W}$ signal. The $\mathrm{W}$ elements are typical $\mathrm{W}^{4+}$ profile at $33.3,35.7$ and $39.7 \mathrm{eV}$ and are assigned to the $\mathrm{W} 4 f_{7 / 2}, \mathrm{~W} 4 f_{5 / 2}$ and $\mathrm{W} 5 p_{3 / 2}$ states [31]. The high-resolution spectrum of the $S 2 p$, characteristic of the $\mathrm{S}^{2-}$ species, is observed at 161.78 and $163.03 \mathrm{eV}$ and is ascribed to the $\mathrm{S} 2 p_{3 / 2}$ and $\mathrm{S} 2 p_{1 / 2}$, respectively [32]. The XRD and XPS

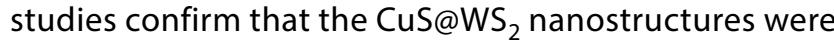
successfully grown on the Ni foam surface.

The energy storage performance of the CuS, $\mathrm{WS}_{2}$ and CuS@WS 2 was analyzed by CV, GCD and EIS measurements. The electrochemical properties of the CuS@WS were tested using three-electrode setup in $3 \mathrm{M} \mathrm{KOH}$ electrolyte. The $\mathrm{KOH}$ electrolyte was found to have very high ionic conductivity; hence, it is the most widely used electrolyte for supercapacitor applications [33]. In the three-electrode setup, platinum wire was selected as the counter electrode, $\mathrm{Ag} / \mathrm{AgCl}$ as the reference electrode and the Ni foam coated with CuS@WS, CuS and WS 2 as the working electrodes at various conditions. Figure $5 \mathrm{a}$ shows the comparative CV curves of CuS, WS 2 and CuS@ 

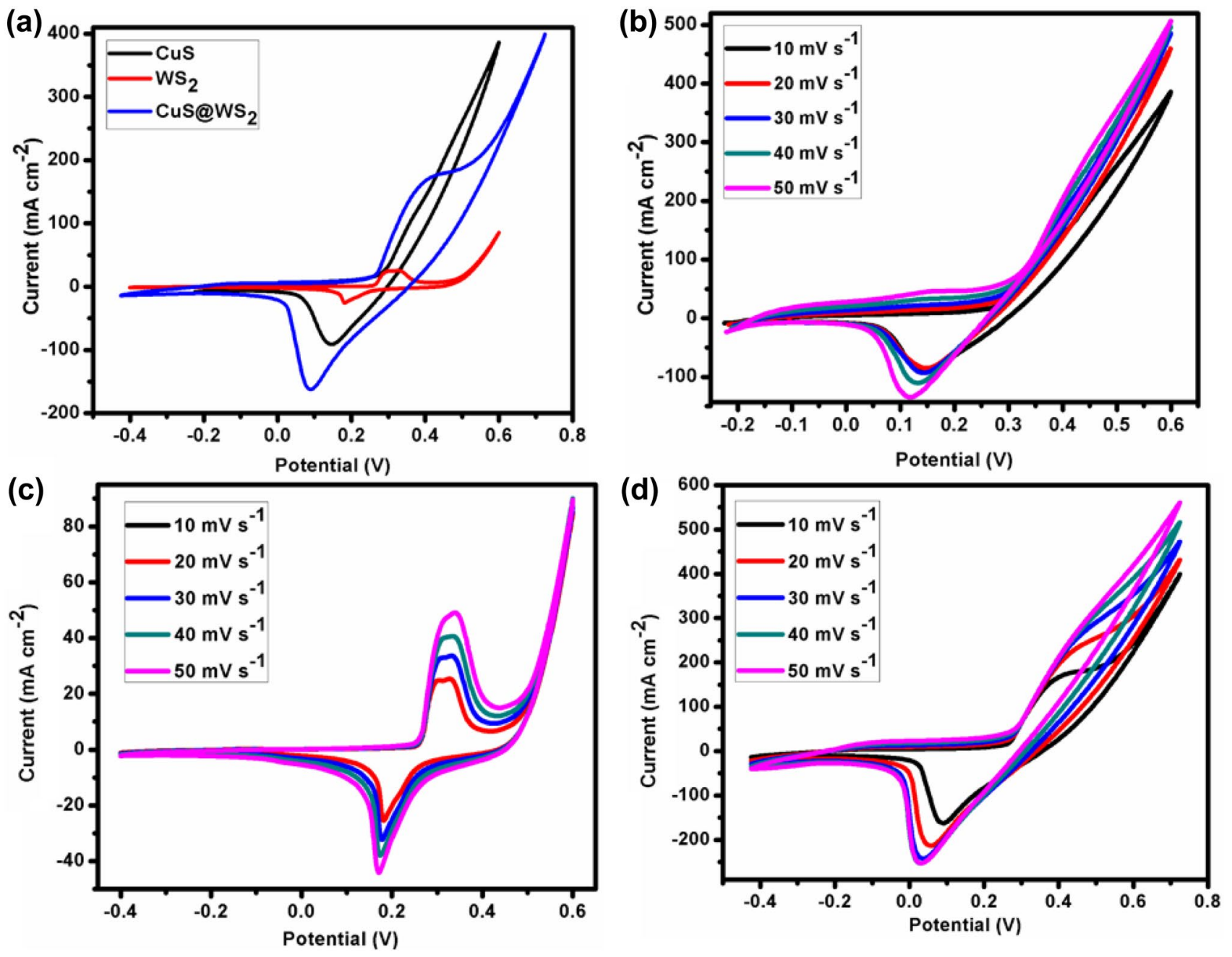

Fig. 5 a Comparison CV curves of the CuS, WS ${ }_{2}$ and CuS@WS $S_{2}$ electrodes at the scan rate of $10 \mathrm{mV} \mathrm{s}^{-1}$. CV curves of the b CuS, c WS ${ }_{2}$ d CuS@WS 2 electrodes at different scan rates of $10-50 \mathrm{mV} \mathrm{s}^{-1}$ in $3 \mathrm{M} \mathrm{KOH}$ solution

$\mathrm{WS}_{2}$ electrodes at a constant scan rate of $10 \mathrm{mV} \mathrm{s}^{-1}$. Interestingly, the CuS@WS ${ }_{2}$ electrode exhibits the higher potential window range of -0.4 to $+0.7 \mathrm{~V}$, which is larger than the CuS $(-0.4$ to $+0.6 \mathrm{~V})$ and $\mathrm{WS}_{2}$ electrode $(-0.4$ to $+0.6 \mathrm{~V})$. As shown in Fig. 5 a, all the electrodes exhibit a pair of redox peaks, which might be attributed to reversible Faradaic redox reactions. Obviously, CuS@WS 2 electrode achieves the larger integration than that of CuS and $\mathrm{WS}_{2}$ electrodes, which is attributed to the synergistic effect of redox reactions associated with $\mathrm{CuS}$ and $\mathrm{WS}_{2}$ materials in composite electrode, thereby achieving superior energy storage performance. Moreover, CV tests were conducted for CuS, WS 2 and CuS@WS electrodes at various scan rates of $10-50 \mathrm{mV} \mathrm{s}^{-1}$ and the corresponding CV profiles are shown in Fig. 5b-d. With the increase in scan rate, the peak current increases and also exhibits the similar CV shape with redox peaks, which indicate that the capacitance characteristics are mainly governed by Faradaic redox reactions. Furthermore, the anodic peak in positive direction and cathodic peak in negative direction were shifted with increasing the scan rate because the charge transfer kinetics limits the reaction [34]. From the following CV curves, composite CuS@WS 2 electrode was found to be the excellent energy storage material when compared to the $\mathrm{CuS}$ and $\mathrm{WS}_{2}$ electrodes.

In addition, galvanostatic charge/discharge (GCD) measurements were conducted to examine the charge storage performance of the as-prepared electrodes. Figure 6 a shows the GCD plots of as-developed $\mathrm{CuS}_{2} \mathrm{WS}_{2}$ and

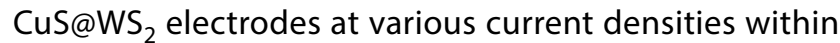
the potential window of 0 and $+0.4 \mathrm{~V}$. Based on GCD plots and Eq. (1), the specific capacitance values of the CuS@ $\mathrm{WS}_{2}$ composite, CuS and $\mathrm{WS}_{2}$ are illustrated in the following table.

The specific capacitance decreased gradually with increasing current density from 3.75 to $15.0 \mathrm{~A} \mathrm{~g}^{-1}$ due to 

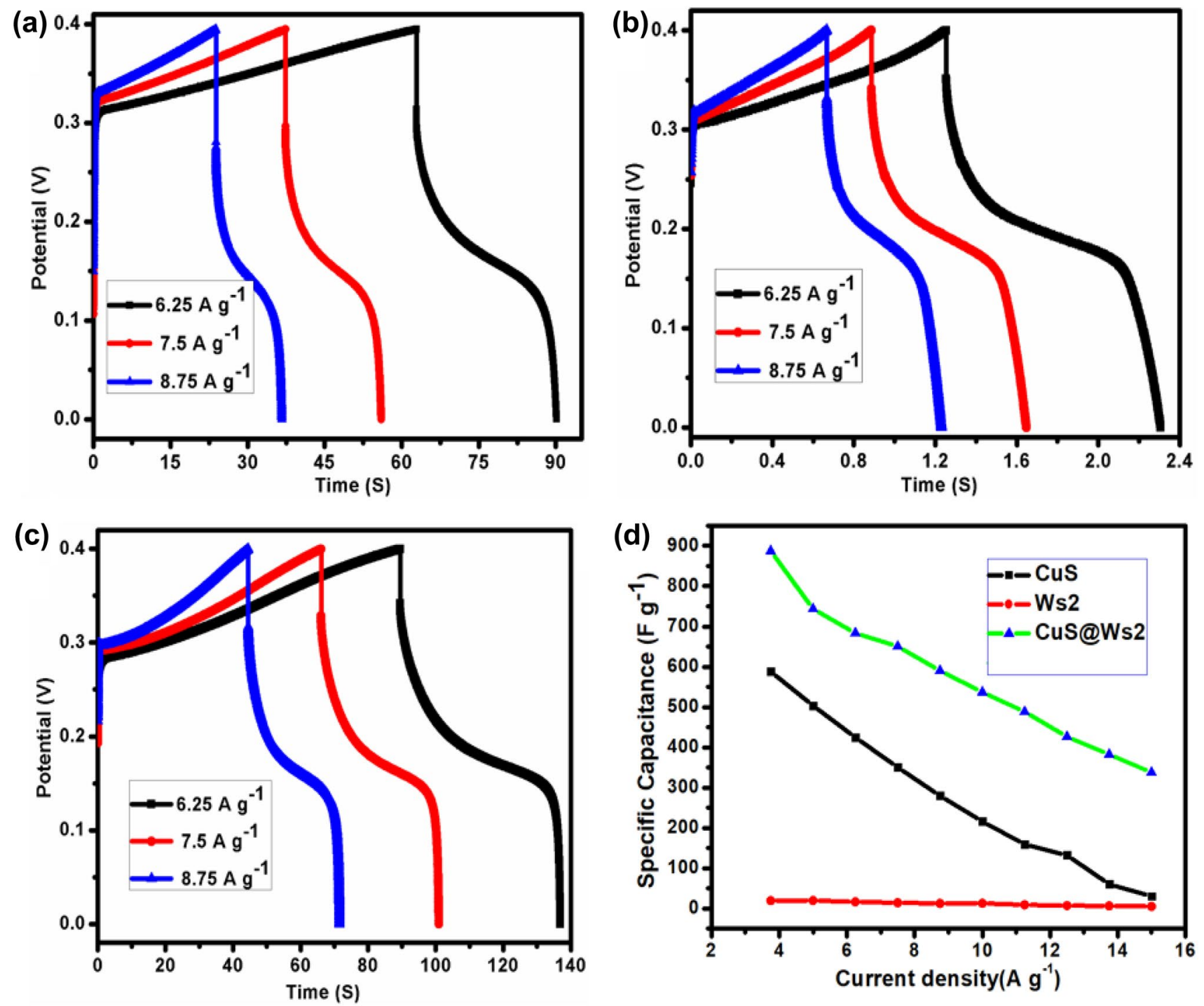

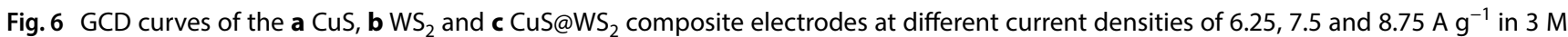
$\mathrm{KOH}$ solution. $\mathbf{d}$ Specific capacitance as a function of current density

the resistance of the electrode and insufficient Faradaic redox reaction at high current densities. The higher specific capacitance of CuS@WS 2 was attributed to its higher surface area with the improved surface morphology of nanostructures [35] (Tables 1, 2).

The energy density and power density are the two important parameters for supercapacitors in real-time applications. Based on the as-obtained specific capacitance values, the energy and power densities of the CuS, WS and CuS@WS $_{2}$ electrodes were calculated and the resulting Ragone plot is shown in Fig. 7. The energy

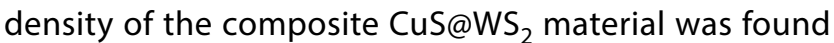
to be $19.16 \mathrm{~W} \mathrm{~h} \mathrm{~kg}^{-1}$ at a power density of $750 \mathrm{~W} \mathrm{~h} \mathrm{~kg}^{-1}$, which is much higher than the CuS and WS $\mathrm{W}_{2}$ electrodes, respectively. Consequently, we concluded that the CuS@

Table 1 Specific capacitance values of the CuS, WS 2 and CuS@WS

\begin{tabular}{llll}
\hline $\begin{array}{l}\text { Current density } \\
(\mathrm{A} / \mathrm{g})\end{array}$ & \multicolumn{3}{l}{ Specific capacitance $(\mathrm{F} / \mathrm{g})$} \\
\cline { 2 - 4 } & CUS & $\mathrm{WS}_{2}$ & CuS@ WS 2 \\
\hline 3.75 & 588.0 & 19.40 & 887.15 \\
5.0 & 536.2 & 19.5 & 683.37 \\
6.25 & 424.37 & 16.40 & 743.59 \\
7.5 & 350.25 & 14.06 & 650.43 \\
8.75 & 279.56 & 12.46 & 590.18 \\
10.0 & 216.0 & 12.75 & 536.75 \\
11.25 & 158.90 & 9.0 & 488.25 \\
12.5 & 132.18 & 7.18 & 426.25 \\
13.75 & 60.15 & 6.18 & 382.25 \\
15.0 & 30.37 & 5.25 & 337.87
\end{tabular}


Table 2 Comparison table of recently reported similar works to CuS@WS

\begin{tabular}{llll}
\hline Material & Capacitance & Current density & References \\
\hline Ti3C2/CuS & $169.5 \mathrm{C} \mathrm{g}^{-1}$ & $1 \mathrm{~A} \mathrm{~g}^{-1}$ & {$[25]$} \\
$\mathrm{CuS} / \mathrm{Cu}(\mathrm{OH})_{2}$ & $845.5 \mathrm{~F} / \mathrm{g}$ & $1 \mathrm{~mA} / \mathrm{cm}^{2}$ & {$[26]$} \\
h-CuS & $83 \mathrm{~m}^{2} \mathrm{~g}^{-1}$ & - & {$[36]$} \\
CuS & $597 \mathrm{~F} \mathrm{~g}^{-1}$ & $1 \mathrm{Ag}^{-1}$ & {$[37]$} \\
CuS@WS & $887.15 \mathrm{~F} \mathrm{~g}_{2}^{-1}$ & $1 \mathrm{~A} \mathrm{~g}^{-1}$ & This work \\
\hline
\end{tabular}

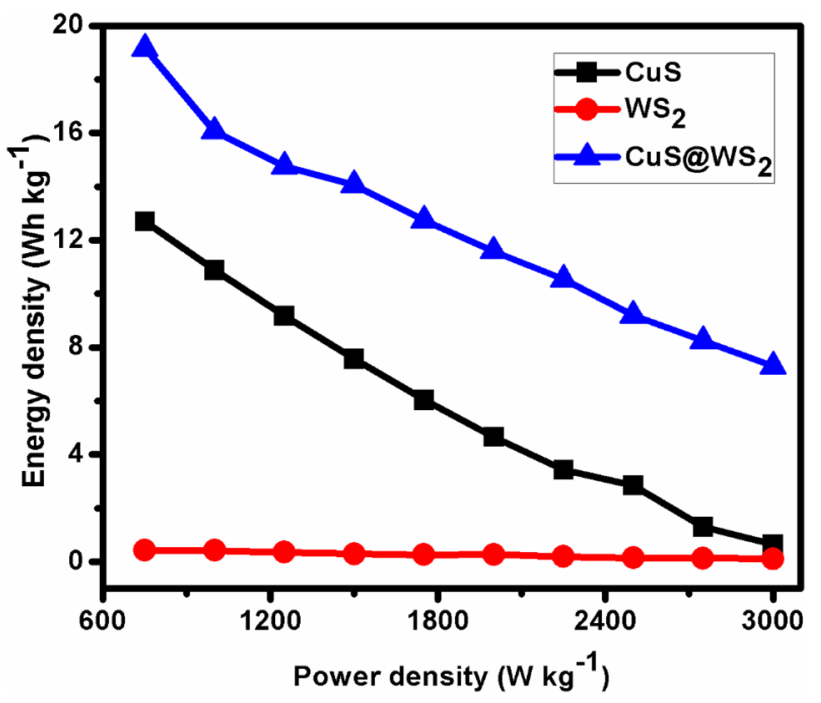

Fig.7 Ragone plots of the CuS-, WS $2^{-}$and CuS@WS 2 -based electrodes

$\mathrm{WS}_{2}$ exhibited a good energy density and power density in supercapacitor applications.

\section{Conclusion}

In summary, the synthesis of nanorice-like structured CuS@WS ${ }_{2}$ nanoparticles was successfully prepared in $3 \mathrm{M}$ $\mathrm{KOH}$ electrolyte in the three-electrode system by using a facile chemical bath deposition method. In comparison with other electrodes, such as CuS and WS , the CuS@WS $_{2}$ electrode showed excellent electrochemical properties including specific capacitance of $887.15 \mathrm{~F} \mathrm{~g}^{-1}$ at $1 \mathrm{~A} \mathrm{~g}^{-1}$. The energy density of the composite CuS@WS 2 material was found to be $19.16 \mathrm{~W} \mathrm{~h} \mathrm{~kg}^{-1}$ at a power density of $750 \mathrm{~W} \mathrm{~h} \mathrm{~kg}^{-1}$, which is much higher than the CuS and WS electrodes, respectively. Since there are a huge number of Faradaic reaction active locations in the contact area with the electrolyte, the CuS@WS 2 electrode supports faster redox reactions and hence it delivers an excellent electrochemical behavior. Therefore, from the detailed analysis, the CuS@WS $\mathrm{W}_{2}$ electrode referred to as a batterytype electrode material can be a potential electrode for high-performance supercapacitor applications.

Acknowledgements This research was supported by Basic Research Laboratory through the National Research Foundations of Korea funded by the Ministry of Science, ICT and Future Planning (NRF2015R1A4A1041584). We also thankful to KBSI for measurements.

\section{Compliance with ethical standards}

Conflict of interest The authors declare that they have no competing interests.

\section{References}

1. Simon P, Gogotsi Y (2008) Materials for electrochemical capacitors. Nat Mater 7:845-854

2. Miller JR, Simon P (2008) Electrochemical capacitors for energy management. Science 321:651-652

3. Reddy AE, Anitha T, Muralee Gopi CVV, Srinivasa Rao S, Kim HJ (2018) NiMoO4@NiWO4 honeycombs as a high-performance electrode material for supercapacitor applications. Dalton Trans 47:9057-9063

4. Winter M, Brodd RJ (2004) What are batteries, fuel cells, and supercapacitors? Chem Rev 104:4245-4270

5. Zhang LL, Zhao XS (2009) Carbon-based materials as supercapacitor electrodes. Chem Soc Rev 38:2520-2531

6. Gopi CVVM, Ravi S, Rao SS, Reddy AE, Kim HJ (2017) Carbon nanotube/metal-sulfide composite flexible electrodes for highperformance quantum dot-sensitized solar cells and supercapacitors. Sci Rep 7:46519

7. Guo T, Yao MS, Lin YH, Nan CW (2015) A comprehensive review on synthesis methods for transition-metal oxide nanostructures. CrystEngComm 17:3551-3585

8. Durga IK, Rao SS, Reddy AE, Gopi CVVM, Kim HJ (2018) Achieving copper sulfide leaf like nanostructure electrode for high performance supercapacitor and quantum-dot sensitized solar cells. Appl Surf Sci 435:666-675

9. Bryan AM, Santino LM, Lu Y, Acharya S, D'Arcy JM (2016) Conducting polymers for pseudocapacitive energy storage. Chem Mater 28:5989-5998

10. Li X, Xue H, Pang H (2017) Facile synthesis and shape evolution of well-defined phosphotungstic acid potassium nanocrystals as a highly efficient visible-light-driven photocatalyst. Nanoscale 9:216-222

11. Sun W, Gao G, Du Y, Zhang K, Wu G (2018) A facile strategy for fabricating hierarchical nanocomposites of $\mathrm{V} 2 \mathrm{O} 5$ nanowire arrays on a three-dimensional $\mathrm{N}$-doped graphene aerogel with a synergistic effect for supercapacitors. J Mater Chem A 6:9938-9947

12. Sun W, Gao G, Zhang K, Liu Y, Wu G (2018) Self-assembled 3D $\mathrm{N}-\mathrm{CNFs} / \mathrm{V} 2 \mathrm{O} 5$ aerogels with core/shell nanostructures through vacancies control and seeds growth as an outstanding supercapacitor electrode material. Carbon 132:667-677

13. Durga IK, Rao SS, Jagadeesh M, Reddy AE, Anitha T, Kim HJ (2018) Synthesis of nanostructured metal sulfides via a hydrothermal method and their use as an electrode material for supercapacitors. New J Chem 42:19183-19192

14. Rui X, Tan H, Yan Q (2014) Nanostructured metal sulfides for energy storage. Nanoscale 6:9889-9924 
15. Xing JC, Zhu YL, Zhou QW, Zheng XD, Jiao QJ (2014) Fabrication and shape evolution of CoS2 octahedrons for application in supercapacitors. Electrochim Acta 136:550-556

16. Krishnamoorthy K, Veerasubramani GK, Radhakrishnan S, Kim SJ (2014) Supercapacitive properties of hydrothermally synthesized sphere like $\mathrm{MoS}_{2}$ nanostructures. Chem Eng J 251:116-122

17. Guo J, Zhang X, Sun Y, Zhang X, Tang L, Zhang X (2017) Doubleshell CuS nanocages as advanced supercapacitor electrode materials. J Power Sour 35:31-35

18. Braga D, Gutiérrez Lezama I, Berger H, Morpurgo AF (2012) Quantitative determination of the band gap of $\mathrm{WS}_{2}$ with ambipolar ionic liquid-gated transistors. Nano Lett 12:5218-5223

19. Rao CNR, Sood AK, Subrahmanyam KS, Govindaraj A (2009) Graphene: the new two-dimensional nanomaterial. Angew Chem Int Ed 48:7752-7777

20. Georgiou T, Jalil R, Belle BD, Britnell L, Gorbachev RV, Morozov SV, Kim YJ, Gholinia A, Haigh SJ, Makarovsky O (2013) Large current modulation in exfoliated-graphene/ $\mathrm{MoS}_{2} /$ metal vertical heterostructures. Nat Nanotechnol 8:100-103

21. Shen L, Yu L, Wu HB, Yu XY, Zhang X, Lou XW (2015) Formation of nickel cobalt sulfide ball-in-ball hollow spheres with enhanced electrochemical pseudocapacitive properties. Nat Commun 6:6694-6702

22. Rao CNR, Gopalakrishnan K, Maitra U, Appl ACS (2015) Comparative study of potential applications of graphene, $\mathrm{MoS}_{2}$, and other two-dimensional materials in energy devices, sensors, and related areas. Mater Interfaces 7:7809-7832

23. Gopalakrishnan K, Sultan S, Govindaraj A, Rao CNR (2015) Supercapacitors based on composites of PANI with nanosheets of nitrogen doped RGO, $\mathrm{BC}_{1.5} \mathrm{~N}, \mathrm{MoS}_{2}$ and $\mathrm{WS}_{2}$. Nano Energy 12:52-58

24. Tu CC, Lin LY, Xiao BC, Chen YS (2016) Highly efficient supercapacitor electrode with two-dimensional tungsten disulfide and reduced graphene oxide hybrid nanosheets. J Power Sour 320:78-85

25. Pan Z, Cao F, Hu X, Ji X (2019) A facile method for synthesizing CuS decorated Ti3C2 MXene with enhanced performance for asymmetric supercapacitors. J Mater Chem A 7:8984-8992

26. Naveen Kumar P, Kalaignan GP, Arulmani S, Anandan S (2018) Solvothermal synthesis of $\mathrm{CuS} / \mathrm{Cu}(\mathrm{OH})_{2}$ nanocomposite electrode materials for supercapacitor applications. J Mater Sci 29:16853-16863

27. Raghavendra KVG, Gopi CVVM, Vinodh R, Durga IK, Kim HJ (2020) One-step facile synthesis of dense cloud-like tiny bundled nanoparticles of CuS nanostructures as an efficient electrode material for high-performance supercapacitors. J Energy Storage 27:101148
28. Himasree P, Durga IK, Krishna TNV, Rao SS, Gopi CVVM, Revathi S, Prabhakar K, Kim HJ (2019) One-step hydrothermal synthesis of CuS@MnS on Ni foam for high performance supercapacitor electrode material. Electrochim Acta 305:467-473

29. Nagaraju C, Gopi CVVM, Ahn JW, Kim HJ (2018) Hydrothermal synthesis of $\mathrm{MoS}_{2}$ and $\mathrm{WS}_{2}$ nanoparticles for high-performance supercapacitor applications. New J Chem 42:12357-12360

30. Durga IK, Rao SS, Ahn JW, Park TY, Bak JS, In-Ho C, Prabhakar K, Kim HJ (2018) Dice-like nanostructure of a CuS@PbS composite for high-performance supercapacitor electrode applications. Energies 11:1624-1635

31. Lim YV, Wang Y, Kong D, Guo L, Wong Jl, Ang LK, Yang HY (2017) Cubic-shaped WS2 nanopetals on a Prussian blue derived nitrogen-doped carbon nanoporous framework for high performance sodium-ion batteries. J Mater Chem A 5:10406-10415

32. Atkin P, Daeneke T, Wang Y, Carey BJ, Berean KJ, Clark RM, Ou JZ, Trinchi A, Cole IS, Kalantarzadeh K (2016) 2D WS 2 /carbon dot hybrids with enhanced photocatalytic activity. J Mater Chem A 4:13563-13571

33. Kim HJ, Kim JH, Kumar CSSP, Punnoose D, Kim SK, Gopi CVVM, Rao SS (2015) An innovative catalyst design as an efficient electro catalyst and its applications in quantum-dot sensitized solar cells and the oxygen reduction reaction for fuel cells. J Electroanal Chem 739:20-27

34. Rao SS, Durga IK, Naresh B, Bak JS, Krishna TNV, In-Ho C, Ahn JW, Kim HJ (2018) One-pot hydrothermal synthesis of novel Cu-MnS with PVP cabbage-like nanostructures for high-performance supercapacitors. Energies 11:1590-1604

35. Himanshu, Rao SS, Punnoose D, Satishkumar P, Gopi CVVM, Naresh B, Durga IK, Krishna TNV, Kim HJ (2018) Development of novel and ultra-high-performance supercapacitor based on a four layered unique structure. Electronics 7:121-135

36. Patil AM, Lokhande AC, Shinde PA, Lokhande CD (2018) Flexible asymmetric solid-state supercapacitors by highly efficient $3 \mathrm{D}$ nanostructured $\mathrm{a}-\mathrm{MnO}_{2}$ and $\mathrm{h}-\mathrm{CuS}$ electrodes. ACS Appl Mater Interfaces 10:16336-16649

37. Peng H, Ma G, Mu J, Sun K, Lei Z (2014) Controllable synthesis of CuS with hierarchical structures via a surfactant-free method for high-performance supercapacitors. Mater Lett 122:25-28

Publisher's Note Springer Nature remains neutral with regard to jurisdictional claims in published maps and institutional affiliations. 